\title{
Optimising diagnostic accuracy with the exercise ECG: opportunities for women and men with stable ischaemic heart disease
}

\author{
Leslee J Shaw, ${ }^{1}$ Joe X Xie, ${ }^{1}$ Lawrence M Phillips, ${ }^{2}$ Abhinav Goyal, ${ }^{1}$ \\ Harmony R Reynolds, ${ }^{2}$ Daniel S Berman, ${ }^{3}$ Michael H Picard, ${ }^{4}$ Balram Bhargava, ${ }^{5}$ \\ Gerard Devlin, ${ }^{6}$ Bernard R Chaitman ${ }^{7}$
}

${ }^{1}$ Department of Medicine, Division of Cardiology, Emory University School of Medicine, Atlanta, Georgia, USA ${ }^{2}$ Cardiovascular Clinical Research Center, Leon $\mathrm{H}$. Charney Division of Cardiology, Department of Medicine, New York University School of Medicine, New York, NY, USA ${ }^{3}$ Department of Imaging, Cedars-Sinai Heart Institute, Cedars-Sinai Medical Center Los Angeles, California, USA ${ }^{4}$ Department of Medicine, Division of Cardiology, Massachusetts General Hospital, Boston, Massachusetts, USA

${ }^{5}$ Professor of Cardiology, Cardiothoracic Sciences Centre, and Executive Director, Stanford India Biodesign Centre, School of International Biodesign (SIB), All India Institute of Medical Sciences, New Delhi, India ${ }^{6}$ Department of Medicine, Division of Cardiology, New Zealand Heart Foundation, Waikato Hospital, Hamilton, New Zealand, USA ${ }^{7}$ Department of Medicine, Division of Cardiology, St Louis University School of Medicine, St Louis, Missouri, USA

\section{Correspondence to} Dr Leslee J Shaw, Room 529, 1462 Clifton Rd NE, Emory University School of Medicine, Atlanta, GA 30322, USA; Ishaw3@emory.edu

Received 18 February 2016 Accepted 28 April 2016

\section{CrossMark}

To cite: Shaw LJ, Xie JX Phillips LM, et al. Heart Asia 2016:8:1-7. doi:10.1136/ heartasia-2016-010736

\section{ABSTRACT}

The exercise ECG is an integral part within the evaluation algorithm for diagnosis and risk stratification of patients with stable ischaemic heart disease (SIHD). There is evidence, both older and new, that the exercise ECG can be an effective and cost-efficient option for patients capable of performing at maximal levels of exercise with suitable resting ECG findings. In this review, we will highlight the major dilemmas in interpreting suspected coronary artery disease symptoms in women and identify optimal strategies for employing exercise ECG as a first-line diagnostic test in the SIHD evaluation algorithm. We will highlight current evidence as well as recent guideline statements on this subject. Trial registration number NCT01471522; Pre-results.

In today's practice of cardiovascular medicine, diverse technology is available to aid diagnosis and risk stratification of patients with stable ischaemic heart disease (SIHD) that include those with suspected and known obstructive coronary artery disease (CAD). These patients represent the core of eligible patients who may be referred for a diagnostic evaluation using exercise ECG or a CAD noninvasive imaging modality. ${ }^{1}$ Although for many physicians there is a perception that all patients require some form of imaging to diagnose CAD, there is evidence, both old and new, that the exercise ECG can be effective and cost-efficient for patients capable of performing at maximal levels of exercise with suitable resting ECG findings. ${ }^{2}$

Our focus on the diagnostic evaluation of women with SIHD is important as there are common misperceptions that the exercise ECG is inaccurate in women and that alternative approaches should be employed to a greater extent, including non-invasive imaging and invasive coronary angiography. Important considerations for the exercise ECG are the abundant evidence on prognostication and diagnostic accuracy in women and its comparative effectiveness compared with other modalities such as nuclear imaging. ${ }^{2}$ In this review, we will highlight the major dilemmas in interpreting suspected $C A D$ symptoms in women and identify optimal strategies for employing exercise ECG as a first-line diagnostic test in the SIHD evaluation algorithm. We will highlight current evidence as well as a recent expert consensus statement from the American Heart Association (AHA) on this subject. ${ }^{3}$

\section{DIAGNOSING CHEST PAIN IN WOMEN}

There are many challenges to diagnosing angina in women; most prominent of which is the reduced prevalence of obstructive CAD in those with chest pain syndromes. ${ }^{4}$ Among any patient subset, there is considerable uncertainty in diagnosing angina even when a diagnostic test is abnormal, when the underlying prevalence of obstructive CAD is relatively low. For physicians, there are several general considerations that may be helpful; a thorough review of this topic was recently published in an AHA expert consensus statement. ${ }^{3}$ First, women generally are more likely to be obese and have a higher prevalence of diabetes. Decreased exercise performance in this type of patient (women and men alike) may reduce the likelihood of completing a maximal test and limit the ability to adequately test coronary reserve. This is seen in both genders; however, due to the higher prevalence of these risk factors in women, the impact on diagnosing obstructive CAD is amplified. Both women and men with orthopaedic limitations or peripheral arterial disease should also be placed into this category of patients who are unlikely to elicit typical angina symptoms due to the patient's decreased ability to reach functional levels where these symptoms would occur. In patients with restriction of physical activity, other forms of symptom provocation, including stress and anxiety, often become more important. Physicians should have a broader view of symptoms in women as it has been well documented that they generally have less typical angina components to their symptom burden. ${ }^{3}$ Importantly, nearly half of women also report dyspnoea on exertion as well as chest pain as presenting symptoms. ${ }^{5-8}$ Based on the Seattle Angina Questionnaire, ${ }^{9}{ }^{10}$ a typical female pattern may be more frequent symptoms with common elements of instability coupled with a lower quality of life, ${ }^{10}$ even for women younger than 55 years of age. ${ }^{11}$ Specific to women, unique prodromal symptoms prior to an acute myocardial infarction (MI), which are less common in men, have been identified and include unusual fatigue, sleep disturbance, as well as dyspnoea. ${ }^{12}$

Understanding what a patient does during everyday life is a fundamental component of optimal decision-making with regard to estimating an individual's capability to perform an adequate exercise test. Women may be more likely than men to subconsciously hide their symptoms of angina by 
revising and accommodating their daily work tasks to avoid activities that bring about symptoms. Thus, simply asking a woman if she is able to do her usual activities may fail to elicit a history of reduced physical work capacity. A woman or man capable of performing usual household activities and a modicum of walking everyday generally has at least a 4-5 metabolic equivalent (MET) work capacity. If a patient engages in more rigorous physical activity, then their MET capacity would be expected to be greater than this.

A greater understanding of the differences in symptom characteristics and the physical abilities between cohorts of women and men with possible symptoms of SIHD can aid referral practice. Ultimately, the practitioner must consider the individual's symptom profile whether the individual patient is male or female.

\section{OPTIMISING LOW-COST TECHNOLOGY}

The exercise ECG is one of the least costly of our diagnostic tests, and for this reason, optimising its use can be an efficient means to achieve substantive cost savings in the diagnostic evaluation of patients with suspected obstructive CAD. It is not medically necessary or cost-effective to routinely refer all patients with suspected CAD to a stress imaging modality for visualisation of left ventricular wall motion or myocardial perfusion at rest and following stress. In addition, in many parts of Asia, patients do not have access to stress imaging modalities without travelling hundreds of kilometres to large cities. For many patients, a routine diagnostic exercise ECG can be an excellent and often the preferred initial procedure. Understanding this latter statement is critical to eliciting cost savings for a given patient population. The value of the exercise ECG lies in its ability to act as a gatekeeper for more advanced imaging procedures. Cost savings are achieved when a patient has a normal test with average (or greater) exercise tolerance and the ability to reach their target heart rate or higher. This includes a majority of women and men who meet eligibility criteria for exercise ECG. Thus, we would like to introduce the concept of an ECG first-line strategy whereby women and men with mildly positive or indeterminate findings then undergo selective stress imaging for further evaluation. This concept of an ECG-first strategy is supported by the results of a recent randomised clinical trial and is further detailed in the recent AHA consensus statement (figure 1$).^{23}$
CANDIDATE SELECTION FOR THE EXERCISE ECG

We can simplify the criteria for candidate selection for the exercise ECG based on the guidance from the 2012 American College of Cardiology (ACC) clinical practice guideline for SIHD (box 1). ${ }^{1}$ Prior consensus documents did not delineate specific criteria and usually stated that the patient should be able to exercise. ${ }^{13}$ However, more recent clinical practice guidelines have made more definitive statements regarding the characteristics of patients with at least moderate physical functioning, defined as the ability to complete household, gardening or recreational work, successfully performing most activities of daily living and without disabling comorbidity, defined as frailty, advanced age (ie, $\geq 70$ years old), marked obesity, peripheral arterial disease, chronic obstructive lung disease or orthopaedic limitations. ${ }^{1}$ These criteria more clearly define those capable of performing an exercise test.

Additional criteria include some pre-test risk assessment with most guidelines selecting patients with an intermediate pre-test risk for the exercise ECG. ${ }^{1}{ }^{13}$ Most global risk scores poorly categorise risk in non-white populations, younger patients and women; but age remains a prominent characteristic of risk for women and men alike. ${ }^{3}$ Risk in women generally reaches an intermediate level in their sixth decade of life, that is, following menopause. A simplified approach to risk estimation was recently devised in the AHA document, which stated that women in their (1) 50s, (2) 60s and (3) 70s and older should generally be considered as low, intermediate and high risk, respectively. ${ }^{3}$ However, if a woman has multiple CAD risk factors, functional disability or extensive comorbidity, increase their risk by one category. Similarly, if there are no risk factors for $\mathrm{CAD}$, decrease their risk by one category. Using this approach, a 50-year-old woman with two risk factors would then be at an intermediate risk. As another example, a 65 -year-old woman with significant functional disability would be increased to a high-risk category. Additional high-risk patients include those with peripheral arterial disease or those with long-standing but poorly controlled diabetes.

By applying these criteria, women and men with suspected or proven SIHD are good candidates for exercise testing if they are at intermediate risk and physically capable, based on the aboveoutlined approach. A final criterion is that their resting ECG should be interpretable for ischaemia (eg, without left bundle branch block, left ventricular hypertrophy with repolarisation abnormalities, ventricular pacemaker) and without significant
Figure 1 Modified clinical evaluation algorithm based on the American Heart Association consensus statement: role of non-invasive testing of women with suspected ischaemic heart disease. ETT, exercise tolerance test; IHD, ischaemic heart disease.

\section{Index IHD Risk Estimate}

No Resting ST Segment Abnormalities

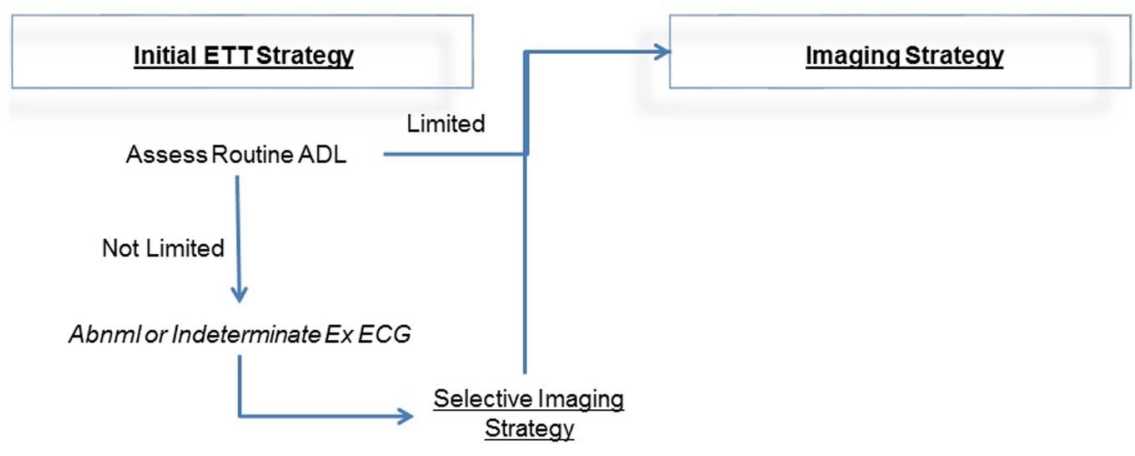


Box 1 Optimal candidate selection for the exercise ECG including symptomatic patients evaluated with stable ischaemic heart disease

- Moderate physical functioning

- Moderate household, yard or recreational work

- Performs most activities of daily living

- Normal resting ECG

- Intermediate likelihood patient

- Women 60 years of age and older

- Women 50-60 years of age with multiple cardiac risk factors

- No disabling comorbidity

- Without frailty

- Not of advanced age (eg, $\geq 70$ years old)

- Not markedly obese

- Without peripheral arterial disease (PAD)

- No chronic obstructive pulmonary disease (COPD)

- No orthopaedic limitations

resting ST-T wave abnormalities (eg, $<1 \mathrm{~mm} \mathrm{ST} \mathrm{depression} \mathrm{at}$ rest). Minor resting ST-T changes are acceptable, but physicians should take care to interpret any exertional changes as calculated by the changes compared with the measured resting values. For example, if a patient has $-0.5 \mathrm{~mm}$ of resting ST segment depression, then their threshold for diagnostic abnormalities during exercise would be a minimum of $\geq-1.5 \mathrm{~mm}$.

Once the decision has been made to refer a patient to exercise testing without imaging, care should be taken to decide on the stress test protocol and the aims for the study. A key aim of the exercise test is for the patient to achieve maximal levels of exercise to avoid stopping the test prior to ischaemia onset as a result of functional limitations. For many women and men, the Bruce protocol is too rigorous with the first stage requiring 4.7 METs of exercise and then graded increases of 2-3 METs every 3 min thereafter. A bicycle protocol with limited incremental increases in watts (eg, 25) per stage may be considered for those patients with limited functional capacity. One way to calculate expected METs is based on a woman's age and is calculated as: $14.7-(0.13 \times$ age $) .{ }^{14}$ Published nomograms on expected MET values are available for women and men. ${ }^{14-16}$ Importantly, the goal is for the protocol to allow the woman or man to achieve maximal levels of exercise or $\geq 85 \%$ predicted maximal heart rate. A major take home point is that identifying the optimal protocol for any given patient is fundamental to getting a good test. Starting with a protocol that increases more gently from 2 to 3 METs and then $\sim 2$ METs per stage may prove beneficial for many women, particularly those of an older age. A modified Bruce (first stage: $1.7 \mathrm{mph}$; $5 \%$ grade) can reduce the challenges with premature fatigue that occur with the standard Bruce protocol. Other protocols, including ramp protocols (increasing 1 MET of exercise every minute), Balke or asymptomatic cardiac ischemia pilot (ACIP), should also be considered for women. Importantly, care should be taken to watch the patient exercise during the first stage of exercise so as to understand how they are handling that initial stage of the test. Excessive dyspnoea or difficulties in walking are signs that the patient may not be able to reach $\geq 85 \%$ predicted maximal heart rate. In some women, there may be a rapid acceleration or an exaggerated increase in their heart rate early on during exercise, such that they may quickly achieve a maximal heart rate. Such rapid increases in heart rate often represent deconditioning. In such patients, additional pharmacological stress imaging may be of value. Finally, it is important to differentiate between premature discontinuation of the test due to functional disability compared with marked ischaemia. The presence of symptoms and concurrent ischaemic ECG changes that occur early during the test are strong signs of $\mathrm{CAD}$ and inadequate coronary blood flow to ischaemic myocardium.

\section{ECG QUALITY IS A MAJOR DETERMINANT OF INTERPRETATIVE CONFIDENCE}

The presence of ST segment changes of $\geq 1 \mathrm{~mm}$ during exercise may be perceived initially as a technically easy measurement to perform. However, there are many challenges to the accurate interpretation of ECG changes. The action of walking on a treadmill can reduce the quality of the ECG tracing. Motion artefact and a wandering baseline can erroneously lead to a conclusion of ischaemia on the ECG, particularly if linked medians are used without attention paid to the raw ECG tracings. A wandering baseline can artificially produce ischaemic-looking ECG abnormalities in the linked median display by 'linking in' baseline drift into the ST segment. In addition, patients have to be reminded not to hold on to the treadmill handrails tightly to avoid artefact resulting from arm muscle contractions. Loose ECG leads can also result in baseline wander, and care should be taken to make sure that lead placement is performed by experienced personnel. Due to these potential issues, it is imperative that interpretation of ST segment changes be made on multiple QRS heartbeats (at least three) instead of relying on a single QRS complex. A typical display would include $2.5 \mathrm{~s}$ of data for three leads with a total of $2.5 \mathrm{~s} /$ lead group for the $10 \mathrm{~s}$ tracing. A way to enhance the reliability of the exercise ECG interpretation is to have a continuous recording of 'raw, unprocessed' leads for the entire $10 \mathrm{~s}$ of recording, for example, leads II, V5 and V6, or to record 'raw ECG data' for $5 \mathrm{~s}$, the precordial leads for $5 \mathrm{~s}$, and then the limb leads for the remaining $5 \mathrm{~s}$. Many treadmill recording systems are also equipped with computer-averaged ECG algorithms that, if analysed without review of the raw data, can lead to false positive results. This could lead to unnecessary downstream testing. The pattern of ST segment changes is an aid to improving the accuracy of interpretation. Generally, but not always, the ST segment changes will continue to worsen during ongoing exercise. In addition to the maximal ST segment changes during exercise, the number of leads involved, the time to onset and offset, and worsening changes in recovery correlate with a greater degree of abnormality of the test. There are patients who elicit ECG changes only in recovery. Such changes may be interpreted similarly as those during exercise, especially if the patient is placed in the supine position post exercise where augmentation of preload may increase myocardial work and elicit ischaemia. The ECG tracing very early in recovery may also help mitigate motion artefact that could accompany peak exercise and aid in the interpretation of the stress ECG. Patients should be monitored for at least five minutes into recovery in order to allow sufficient time for documentation of any recovery ST segment changes.

ST segment changes defining ischaemia come in many forms. Similar to the acute presentation, ST segment elevation of $\geq 1 \mathrm{~mm}$ (at $60-80 \mathrm{~ms}$ after the $\mathrm{j}$ point) in a lead that does not have a $\mathrm{Q}$ wave is an important ischaemic marker and is a sign of severe (usually transmural) ischaemia. Recent evidence supports the utility of ST segment elevation in aVR, particularly if greater than lead V1, as reflective of high-risk CAD, including significant left main CAD, multivessel CAD or proximal left anterior 
descending obstruction. ${ }^{17}{ }^{18}$ Horizontal or downsloping ST segment depression of $\geq 1 \mathrm{~mm}$ (at $60-80 \mathrm{~ms}$ after the $\mathrm{j}$ point) is the traditional definition of an ischaemic ECG response during the exercise test. The timing and magnitude of ST segment depression as well as concurrent exertional symptoms (chest pain or other ischaemic equivalents, such as dyspnoea) are important components to document during the stress test. Although slowly upsloping ST segment depression $>1.5 \mathrm{~mm}$ has historically been less accurate for detection of CAD, when occurring with typical chest pain (ie, requiring exercise termination) it is considered an ischaemic response.

A systematic review of evidence for diagnostic accuracy for the exercise ECG was recently reported. ${ }^{1}{ }^{19}$ As has been widely held in the past, it reveals that in women the diagnostic sensitivity and specificity of $\geq 1 \mathrm{~mm}$ of ST segment depression (horizontal or downsloping) are not high: $62 \%$ and $68 \%$, respectively. ${ }^{19}$ Slightly higher rates have been reported in largely male cohorts undergoing exercise ECG testing. ${ }^{1}$ It is important to recognise, however, that the exercise ECG changes are only one piece of data to be included in the gamut of information available from the exercise stress test.

\section{MORE THAN THE ECG: THE MULTITUDE OF COMPONENTS OF RISK ASSESSMENT FROM THE EXERCISE TEST}

One key to increasing the accuracy and value of the exercise ECG is to integrate multiple parameters observed during testing -beyond ST segment changes. Relying solely on ECG changes will result in reduced diagnostic and prognostic accuracy, particularly for women. ${ }^{20}$ An example of an integrated approach is the similar prognostic accuracy of the Duke treadmill score in women and men. ${ }^{20}$ The Duke treadmill score integrates exercise duration, ST segment changes and exertional chest pain into a numerical value whereby risk categories have been devised for women and men. The score is calculated as: exercise time $(5 \times$ ST deviation $)-(4$ exertional chest pain $) .{ }^{20}$ For this score, exercise time is measured in minutes, ST deviation is the largest measurement in millimetres of either depression or elevation in any lead (except aVR), and exertional chest pain is based on a scale of $0=$ no chest pain during exercise, $1=$ non-limiting chest pain and $2=$ exercise-limiting chest pain. The Duke treadmill score has been shown to effectively risk-stratify both women and men. ${ }^{20}$ Based on the Duke treadmill score, low risk is a score of $\geq+5$, high risk $\leq-11$; with intermediate risk as values in between 4 and -10 . There are other treadmill risk scores including the Lauer score, which incorporates clinical data including risk factors and the Morise score that includes data specific to women including the use of hormone replacement therapy. ${ }^{21} 22$

\section{OTHER PROGNOSTIC MARKERS}

The most prominent risk marker from the exercise test for both women and men is the duration of exercise. There is an inversely proportional relationship between exercise capacity and risk of CAD events, whereby patients with excellent work capacity are at low risk and those with functional disability at high risk. Patients unable to exercise beyond 5 METs of exercise (or stage I of the Bruce protocol) are those considered at higher clinical risk. Generally, patients exercising into Bruce stage III or higher have good-excellent physical work capacity. As women generally have a lower physical work capacity compared with men, we would expect a larger proportion of women to demonstrate physical limitations during exercise testing. From the St James Women Take Heart registry, failure to exercise beyond 5 METs of exercise was associated with the highest mortality. ${ }^{23}$ This would be particularly true for older women where the average exercise duration of a woman aged $\geq 70$ years is 5 METs. ${ }^{14}$

Exertional chest pain, a main component of the Duke treadmill score, can be categorised as present or severe enough to be the reason for stopping the test. Either way, this variable has not been shown to be independently predictive of obstructive CAD or 5 -year IHD mortality in women. ${ }^{20}$ However, if concurrent with ECG or other abnormalities (eg, ventricular arrhythmias), then this symptom should be considered as potentially ischaemic in origin. Care should also be taken to consider excessive dyspnoea on exertion as this may be an ischaemic equivalent. When significant ST segment changes occur during exercise testing in the absence of exertional chest pain, a patient's clinical history of angina may prove useful to interpret the likelihood of CAD.

The presence of arrhythmias during exercise has been controversial as sympathetic stimulation that increases heart rate may also benignly increase the presence of premature ventricular contractions. However, the occurrence of frequent or more complex ventricular arrhythmias, such as ventricular tachycardia or ventricular fibrillation, is an ischaemic, high-risk marker. ${ }^{24-26}$ Some reports have identified $>7$ premature ventricular contractions per minute as indicative of ischaemia. ${ }^{3}$

Although maximal predicted heart rate (MPHR) is generally calculated as 220 - age, there is a female-specific equation, which is $\left(206-0.88\right.$ [Age]) and may be preferred. ${ }^{27}$ During exercise, a patient's heart rate increases with the physical demands of the exercise stage such that there is a graded but linear increase in heart rate during the duration of exercise. While for men a peak exercise of $<80 \%$ of MPHR is generally considered a submaximal test, for women an observed peak exercise heart rate $<85 \%$ is considered submaximal. ${ }^{27}$ Anti-ischaemic and blood pressure medications impact on the heart rate and blood pressure responses to exercise; as such, it is preferable to discontinue anti-ischaemic agents for 24-48 hours prior to testing (if possible). A failure to increase heart rate with increasing levels of exercise or a blunted heart rate response (in a patient not under the influence of beta-blockers) is a sign of severe CAD stenosis, including left main disease, and associated with major CAD event risk. ${ }^{28} 29$ This is defined as impaired chronotropic reserve. Some reports have identified a peak heart rate of $\leq 110$ $120 \mathrm{bpm}$ as signifying high risk; the latter of which would depend on the patient's age and if they had taken any recent anti-ischaemic medications.

At the cessation of exercise, a normal heart rate response is a return towards normal levels within a few minutes into recovery. A persistently elevated heart rate during recovery where reductions from peak levels do not exceed $12-24 \mathrm{bpm}$ is considered abnormal. The underlying rationale is that there is sympathetic activation increasing the heart rate during exercise and a persistently elevated heart rate response represents a failure to restore parasympathetic tone. Heart rate recovery $\leq 12-24 \mathrm{bpm}$ is an additional high-risk marker and has been shown to predict CAD and all-cause mortality. ${ }^{28} 30-32$

A final marker that has been considered is blood pressure response. The normal blood pressure response to graded exercise is an increase in systolic measurements with a delta change in diastolic findings of $\pm 10 \mathrm{~mm} \mathrm{Hg}$. A blunted or even a drop in systolic blood pressure with increasing physical exercise indicates an inability of the ventricle to perform sufficiently for the work intensity. This marker of an impaired blood pressure response to exercise has been associated with a severe stenosis, particularly in the left anterior descending or left main coronary artery, or in the setting of multivessel CAD. ${ }^{33} 34$ 


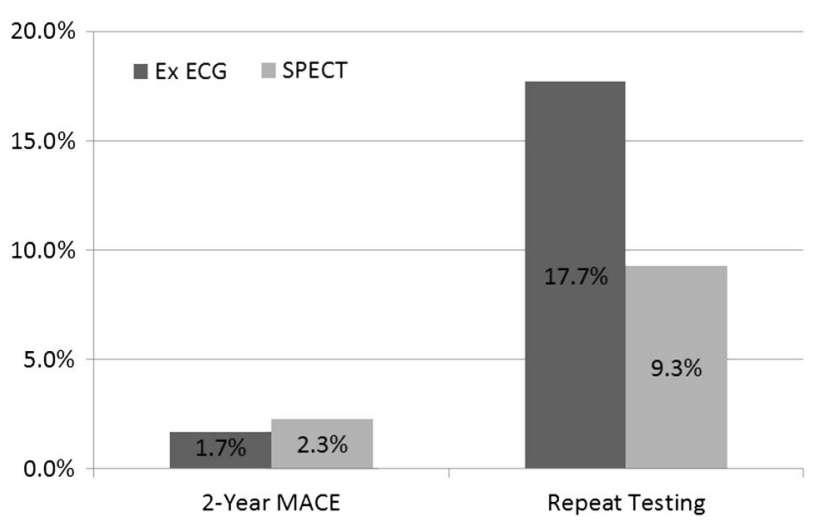

Figure 2 Major findings from the What is the Optimal Method of Ischemia Elucidation in Women? trial reporting similar 2-year rates of major adverse cardiac events (MACE) and a low rate of follow-up testing in women randomised to the exercise ECG (Ex ECG).

\section{COMPARATIVE EFFECTIVENESS OF THE EXERCISE ECG VERSUS MYOCARDIAL PERFUSION SPECT IN WOMEN}

Recently, the What is the Optimal Method of Ischemia Elucidation in Women? (WOMEN) trial $^{2}$ reported similar 2-year outcomes (death, acute coronary syndrome or heart failure hospitalisation) for 824 females randomised to an exercise ECG compared with exercise myocardial perfusion SPECT (figure 2). This trial also reported nearly 50\% cost savings by employing the exercise ECG as the initial procedure with selected follow-up imaging (ie, myocardial perfusion SPECT) in only $18 \%$ of patients. Women randomised to the exercise ECG had exertional ischaemia ruled out and with excellent clinical outcomes over 2 years of follow-up. This trial underscores that low-cost technology, such as the exercise ECG, can adequately diagnose patients and be applied in an optimal and efficient strategy of care for women with SIHD.

\section{WHAT TO DO WITH THE TEST RESULTS}

The total of ECG parameters including ST segment deviation, onset/offset, extent and associated markers for heart rate, blood pressure and arrhythmias encompasses a comprehensive approach to exercise test interpretation. Although the ECG alone may have a diagnostic accuracy in women, which is less than that of their male counterparts (diagnostic sensitivity: 62\%; specificity: 68\%), the incorporation of multiple parameters improves test accuracy and physician certainty in the diagnosis of CAD. ${ }^{13}$ Moreover, assessment of functional capacity and delineating the onset of ischaemia provide tremendous insight into the burden of provocative symptoms in a patient's daily life and guidance to monitoring the effectiveness of anti-ischaemic therapy.

Supportive rationale for employing an exercise ECG as the primary diagnostic procedure is the negative predictive value of the test's findings. For women and men alike, the presence of an adequate level of exertional stress to maximal heart rate values without ischaemic ECG changes or other risk markers is associated with a low likelihood of obstructive CAD and excellent survival. ${ }^{13}$ From a recent trial, nearly two-thirds of women undergoing exercise ECG had a negative maximal stress test. ${ }^{2}$

However, should a patient have inadequate levels of exercise, residual uncertainty remains that the intensity of stress was insufficient to provoke ischaemia. In these cases, the use of stress imaging may aid in further evaluating the patient with a pharmacological stressor (figure 1). This type of secondary evaluation was recently explored in the WOMEN trial. ${ }^{2}$ For the women randomised to an exercise ECG test, only 20\% had abnormal ST segment changes and required additional imaging with myocardial perfusion SPECT. This strategy of an ECG first is important as the majority of women and men will not require additional imaging, particularly in light of good exercise tolerance and a negative ECG response to exercise.

Positive tests should be followed up with medical management for SIHD, both anti-atherosclerotic (eg, statins, aspirin, lifestyle measures) and anti-anginal (eg, beta-adrenergic blockade, calcium channel blockade, nitrates). Invasive coronary angiography may be considered for patients with strongly positive findings. One challenge with the exercise ECG is the inability to quantify the amount of ischaemia and the poor localisation of ST segment alterations as identifying vascular territory involvement. Contrary to stress nuclear imaging where the percentage of ischaemic myocardium has known prognostic significance, the ECG does not have this same capacity to quantify ischaemia. Thus, strongly positive findings are defined based on early
Figure 3 Exercise ECG of a patient screened for the International Study of Comparative Health Effectiveness with Medical \& Invasive Approaches trial. The 12-lead ECG is an example of computer-averaged information (ie, linked median) with the raw data (lead II on the bottom row) indicating marked ECG artefact. By examining the averaged ECG findings without the raw data, the interpretation of ST segment changes can be highly misleading.

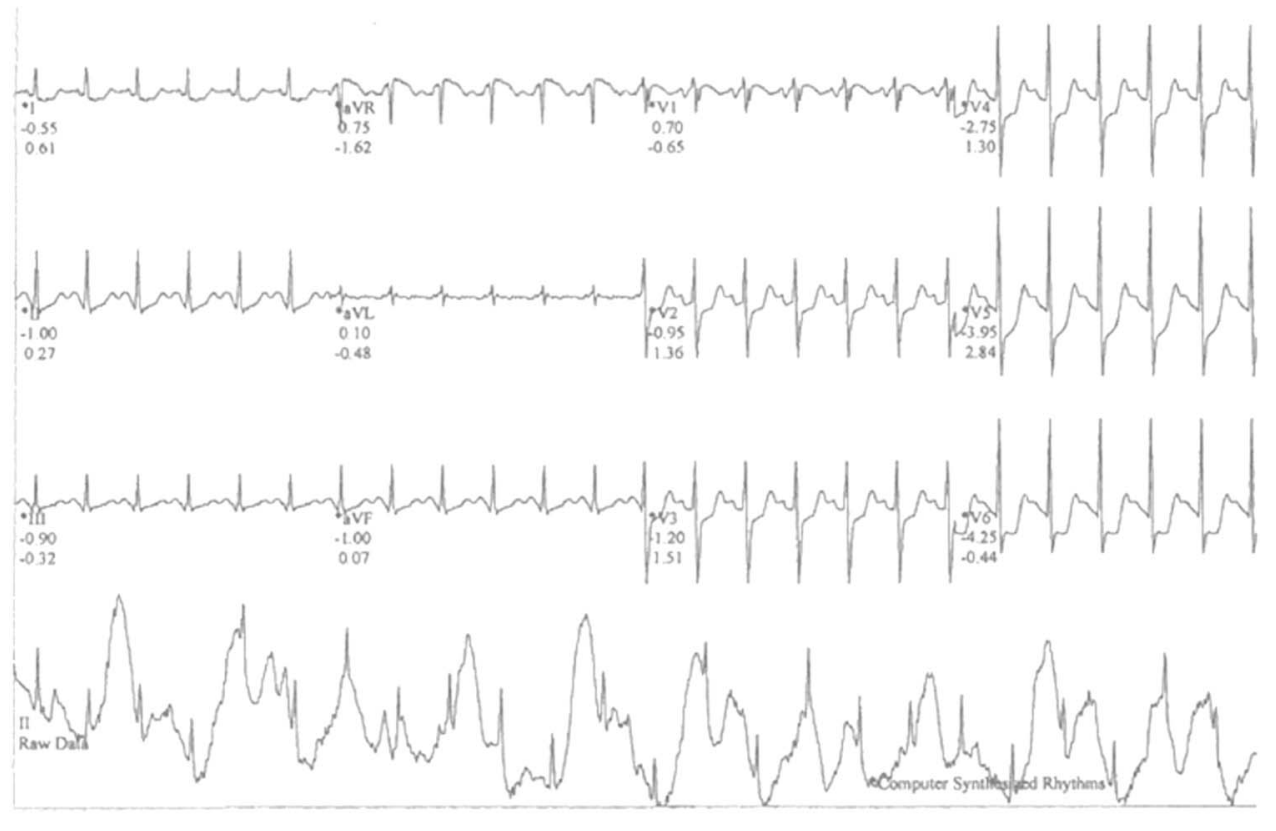




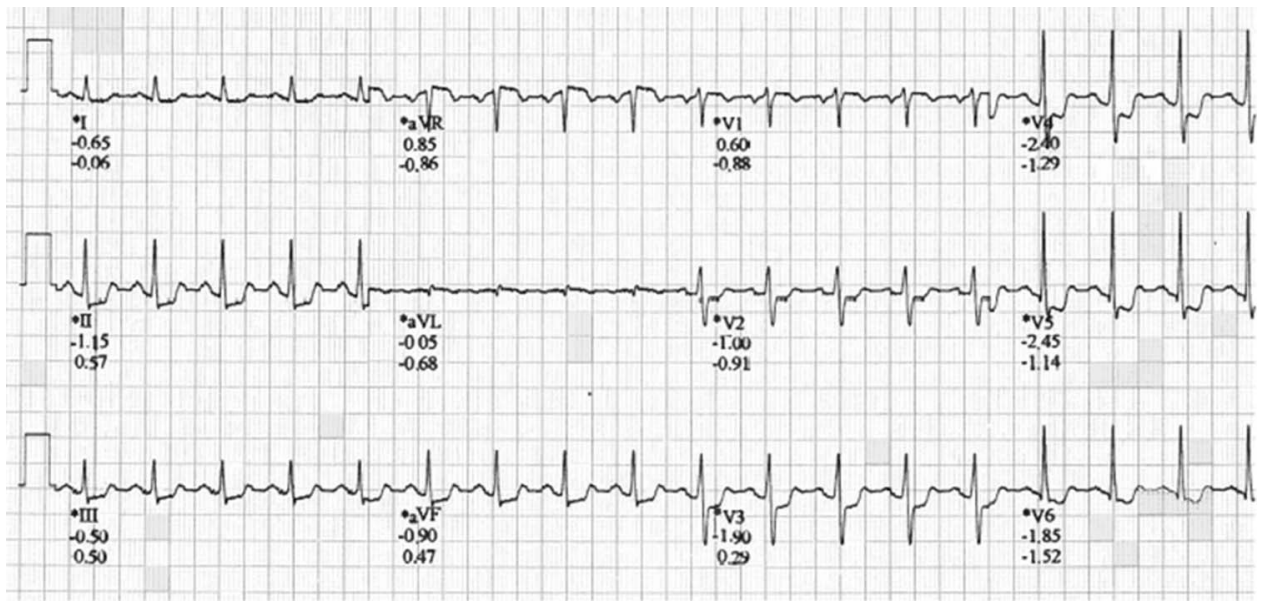

Figure 4 Exercise ECG of a patient with marked ST segment changes at peak exercise. Note the marked horizontal/downsloping inferolateral ST segment depression and prominent ST elevation in lead aVR. This patient qualified for participation in the International Study of Comparative Health Effectiveness with Medical \& Invasive Approaches trial.

onset, number of leads involved, persistence of ECG changes into recovery and concomitant patient symptoms. Despite this, the lack of quantification does hinder patient management and triaging for SIHD care.

\section{RECENT COMPARATIVE EFFECTIVENESS TRIALS OF STRESS TEST STRATEGIES}

The exercise ECG has also been an integral part of other SIHD trials. ${ }^{35} 36$ Based on these trials, current evidence supports that a majority of patients with SIHD may be managed with an initial strategy of optimal medical therapy. ${ }^{35}{ }^{37}$ Several large randomised trials have been published, which established that there is no improvement in outcomes with coronary revascularisation compared with medical management strategies. In the Clinical Outcomes Using Revascularization and Aggressive Drug Evaluation (COURAGE) trial, many patients were randomised following an exercise ECG test with evidence of ischaemia. ${ }^{37} \mathrm{In}$ these patients, similar rates of all-cause mortality or non-fatal MI were observed over 5 years for patients with CAD randomised to medical therapy compared with percutaneous coronary intervention with medical therapy. In both randomised arms, medical therapy was optimised with the effective aim of angina-free status. Trials, such as COURAGE, illustrate the importance and effectiveness of anti-ischaemic therapies as well as the link to ischaemia-provoking tests such as the exercise ECG as fundamental to caring for patients with CAD. ${ }^{38}$ This approach to optimised medical management as a front-line approach to caring for the patient with SIHD is similarly applicable to women and men. ${ }^{36}$

The National Institutes of Health is currently funding a follow-up randomised trial that has numerous enrolling centres in Asia. This ongoing trial, entitled the International Study of Comparative Health Effectiveness with Medical \& Invasive Approaches (ISCHEMIA), is randomising patients with SIHD to an invasive approach compared with medical management. In this trial, patients are eligible based on core laboratorydetermined ECG changes and then patients are screened for CAD with coronary computed tomographic angiography (CCTA). We include a few cases of trial-eligible ischaemia from the ISCHEMIA trial in figures 3 and 4.

\section{SUMMARY}

The exercise ECG has an extensive body of evidence supporting its utility; with more recent evidence documenting its effectiveness in women with SIHD. For many women, a negative ECG in the setting of good exercise capacity and a maximal heart rate response effectively rules out CAD; and this is the primary benefit of the exercise test. Women in this latter category have an excellent long-term outcome. Incorporation of all available data as a multimarker approach to test interpretation improves accuracy. In this regard, integration of symptoms, heart rate, occurrence of arrhythmias, blood pressure response, physical work capacity, as well as ECG changes provides marked improvements in detection of CAD and for risk-stratification purposes. If properly used, the exercise ECG is equally effective and costs less than a stress imaging approach. ${ }^{2}$ This ECG-first strategy may prove beneficial to many women as well as men with SIHD.

Twitter Follow Leslee Shaw at @lesleejshaw

Contributors All authors have contributed to this review in organisation, review inception, writing and critical review of the submitted manuscript.

Competing interests None declared.

Provenance and peer review Not commissioned; externally peer reviewed.

\section{REFERENCES}

1 Fihn SD, Gardin JM, Abrams J, et al. 2012 ACCF/AHA/ACP/AATS/PCNA/SCAI/STS Guideline for the diagnosis and management of patients with stable ischemic heart disease: a report of the American College of Cardiology Foundation/American Heart Association Task Force on Practice Guidelines, and the American College of Physicians, American Association for Thoracic Surgery, Preventive Cardiovascular Nurses Association, Society for Cardiovascular Angiography and Interventions, and Society of Thoracic Surgeons. J Am Coll Cardiol 2012;60:e44-164.

2 Shaw LJ, Mieres JH, Hendel RH, et al. Comparative effectiveness of exercise electrocardiography with or without myocardial perfusion single photon emission computed tomography in women with suspected coronary artery disease: results from the What Is the Optimal Method for Ischemia Evaluation in Women (WOMEN) trial. Circulation 2011;124:1239-49.

3 Mieres JH, Gulati M, Bairey Merz N, et al. Role of noninvasive testing in the clinical evaluation of women with suspected ischemic heart disease: a consensus statement from the American Heart Association. Circulation 2014;130:350-79.

4 Cheng VY, Berman DS, Rozanski A, et al. Performance of the traditional age, sex, and angina typicality-based approach for estimating pretest probability of angiographically significant coronary artery disease in patients undergoing coronary computed tomographic angiography: results from the multinational coronary CT angiography evaluation for clinical outcomes: an international multicenter registry (CONFIRM). Circulation 2011;124:2423-32, 1-8.

5 Kang $X$, Shaw LJ, Hayes SW, et al. Impact of body mass index on cardiac mortality in patients with known or suspected coronary artery disease undergoing myocardial perfusion single-photon emission computed tomography. J Am Coll Cardiol 2006;47:1418-26. 
6 Abidov A, Rozanski A, Hachamovitch R, et al. Prognostic significance of dyspnea in patients referred for cardiac stress testing. N Engl J Med 2005;353:1889-98.

7 Shaw LJ, Hendel RC, Cerquiera M, et al. Ethnic differences in the prognostic value of stress technetium-99m tetrofosmin gated single-photon emission computed tomography myocardial perfusion imaging. J Am Coll Cardiol 2005;45:1494-504.

8 Kay J, Dorbala S, Goyal A, et al. Influence of sex on risk stratification with stress myocardial perfusion Rb-82 positron emission tomography: results from the PET (Positron Emission Tomography) Prognosis Multicenter Registry. J Am Coll Cardiol 2013;62:1866-76.

9 Kimble LP, Dunbar SB, Weintraub WS, et al. The Seattle angina questionnaire: reliability and validity in women with chronic stable angina. Heart Dis 2002:4:206-11.

10 Norris CM, Saunders LD, Ghali WA, et al. Health-related quality of life outcomes of patients with coronary artery disease treated with cardiac surgery, percutaneous coronary intervention or medical management. Can J Cardiol 2004;20:1259-66.

11 Dreyer RP, Wang Y, Strait KM, et al. Gender differences in the trajectory of recovery in health status among young patients with acute myocardial infarction: results from the variation in recovery: role of gender on outcomes of young AMI patients (VIRGO) study. Circulation 2015;131:1971-80.

12 McSweeney JC, Cody M, O'Sullivan P, et al. Women's early warning symptoms of acute myocardial infarction. Circulation 2003;108:2619-23.

13 Mieres JH, Shaw $L$, Arai A, et al. Role of noninvasive testing in the clinical evaluation of women with suspected coronary artery disease: Consensus statement from the Cardiac Imaging Committee, Council on Clinical Cardiology, and the Cardiovascular Imaging and Intervention Committee, Council on Cardiovascular Radiology and Intervention, American Heart Association. Circulation 2005;111:682-96.

14 Gulati M, Black HR, Shaw LJ, et al. The prognostic value of a nomogram for exercise capacity in women. N Engl J Med 2005;353:468-75.

15 Myers J, Prakash $M$, Froelicher $\mathrm{V}$, et al. Exercise capacity and mortality among men referred for exercise testing. N Engl J Med 2002;346:793-801.

16 Morris CK, Myers J, Froelicher VF, et al. Nomogram based on metabolic equivalents and age for assessing aerobic exercise capacity in men. J Am Coll Cardiol 1993;22:175-82.

17 Yamaji H, Iwasaki K, Kusachi S, et al. Prediction of acute left main coronary artery obstruction by 12-lead electrocardiography. ST segment elevation in lead aVR with less ST segment elevation in lead V(1). J Am Coll Cardiol 2001;38:1348-54.

18 Gorgels AP, Engelen DJ, Wellens HJ. Lead aVR, a mostly ignored but very valuable lead in clinical electrocardiography. J Am Coll Cardiol 2001:38:1355-6.

19 http://effectivehealthcare.ahrq.gov/index.cfm/search-for-guides-reviews-and-reports/? productid $=1132$ \&pageaction $=$ displayproduct.

20 Alexander KP, Shaw L, Shaw LK, et al. Value of exercise treadmill testing in women. J Am Coll Cardiol 1998:32:1657-64.

21 Morise AP, Lauer MS, Froelicher VF. Development and validation of a simple exercise test score for use in women with symptoms of suspected coronary artery disease. Am Heart J 2002;144:818-25.
22 Lauer MS, Pothier CE, Magid DJ, et al. An externally validated model for predicting long-term survival after exercise treadmill testing in patients with suspected coronary artery disease and a normal electrocardiogram. Ann Intern Med 2007:147:821-8

23 Gulati M, Pandey DK, Arnsdorf MF, et al. Exercise capacity and the risk of death in women: the St James Women Take Heart Project. Circulation 2003;108: 1554-9.

24 Graboys TB. Import of exercise-induced ventricular arrhythmia in malignant ventricular arrhythmia. Am J Cardiol 1983;51:1570.

25 Pedrosa RC, Salles JH, Magnanini MM, et al. Prognostic value of exercise-induced ventricular arrhythmia in Chagas' heart disease. Pacing Clin Electrophysiol 2011;34:1492-7.

26 Podrid PJ, Bumio F, Fogel RI. Evaluating patients with ventricular arrhythmia. Role of the signal-averaged electrocardiogram, exercise test, ambulatory electrocardiogram, and electrophysiologic studies. Cardiol Clin 1992;10:371-95.

27 Gulati M, Shaw LJ, Thisted RA, et al. Heart rate response to exercise stress testing in asymptomatic women: the St. James women take heart project. Circulation 2010;122:130-7.

28 Lauer MS, Okin PM, Larson MG, et al. Impaired heart rate response to graded exercise. Prognostic implications of chronotropic incompetence in the Framingham Heart Study. Circulation 1996;93:1520-6.

29 Lauer MS. Chronotropic incompetence: ready for prime time. J Am Coll Cardiol 2004:44:431-2.

30 Lauer MS, Froelicher V. Abnormal heart-rate recovery after exercise. Lancet 2002;360:1176-7.

31 Vivekananthan DP, Blackstone EH, Pothier CE, et al. Heart rate recovery after exercise is a predictor of mortality, independent of the angiographic severity of coronary disease. J Am Coll Cardiol 2003:42:831-8.

32 Cheng YJ, Lauer MS, Earnest CP, et al. Heart rate recovery following maximal exercise testing as a predictor of cardiovascular disease and all-cause mortality in men with diabetes. Diabetes Care 2003;26:2052-7.

33 Singh JP, Larson MG, Manolio TA, et al. Blood pressure response during treadmill testing as a risk factor for new-onset hypertension: the Framingham heart study. Circulation 1999;99:1831-6.

34 Lauer MS. Resuscitating the exercise stress test. Cleve Clin J Med 1999;66:278-82.

35 Sedlis SP, Hartigan PM, Teo KK, et al. Effect of PCl on Long-Term Survival in Patients with Stable Ischemic Heart Disease. N Engl J Med 2015;373:1937-46.

36 Boden WE, O'Rourke RA, Teo KK, et al. Impact of optimal medical therapy with or without percutaneous coronary intervention on long-term cardiovascular end points in patients with stable coronary artery disease (from the COURAGE Trial). Am J Cardiol 2009;104:1-4.

37 Boden WE, O'Rourke RA, Teo KK, et al. Optimal medical therapy with or without PCl for stable coronary disease. N Engl I Med 2007;356:1503-16.

38 Stone GW, Hochman JS, Williams DO, et al. Medical Therapy With Versus Without Revascularization in Stable Patients With Moderate and Severe Ischemia: The Case for Community Equipoise. J Am Coll Cardiol 2016;67:81-99. 